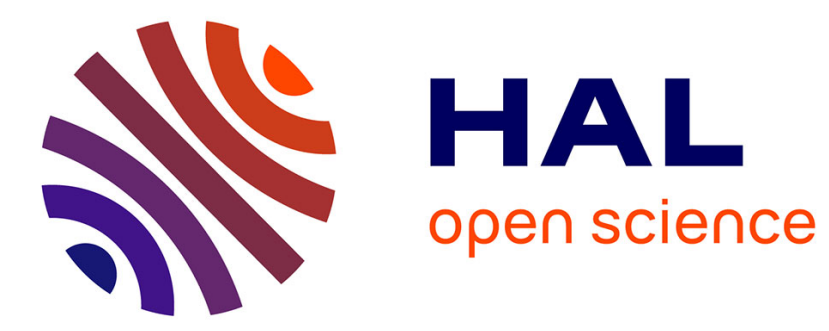

\title{
Exposition aux risques professionnels pendant la grossesse : retour de services médicaux interentreprises
}

Jean-Bernard Henrotin, Monique Vaissière, Maryline Etaix, Mathieu Dziurla, Stéphane Malard, Dominique Lafon

\section{- To cite this version:}

Jean-Bernard Henrotin, Monique Vaissière, Maryline Etaix, Mathieu Dziurla, Stéphane Malard, et al.. Exposition aux risques professionnels pendant la grossesse : retour de services médicaux interentreprises. Gynécologie Obstétrique Fertilité \& Sénologie, 2018, 46 (1), pp.20-27. 10.1016/j.gofs.2017.10.029 . hal-01972390

\section{HAL Id: hal-01972390 \\ https://hal.science/hal-01972390}

Submitted on 7 Jan 2019

HAL is a multi-disciplinary open access archive for the deposit and dissemination of scientific research documents, whether they are published or not. The documents may come from teaching and research institutions in France or abroad, or from public or private research centers.
L'archive ouverte pluridisciplinaire HAL, est destinée au dépôt et à la diffusion de documents scientifiques de niveau recherche, publiés ou non, émanant des établissements d'enseignement et de recherche français ou étrangers, des laboratoires publics ou privés.

\section{(ㅇ)(1) $\$$}

Distributed under a Creative Commons Attribution - NonCommercial - NoDerivatives 44.0 


\title{
Exposition aux risques professionnels pendant la grossesse : retour de services médicaux interentreprises.
}

\author{
Occupational risks during pregnancy: \\ feedback from occupational medical services \\ (suivi des grossesses au travail)
}

\section{Jean-Bernard Henrotin ${ }^{1}$, Monique Vaissière ${ }^{2}$, Maryline Etaix ${ }^{3}$, Mathieu Dziurla ${ }^{1}$, Stéphane Malard ${ }^{4}$ Dominique Lafon $^{5}$}

${ }^{1}$ Département d'épidémiologie en entreprise, Institut National de Recherche et sécurité, Vandœuvre-Les-Nancy, France

2 Service de santé au travail, Santé Travail Béziers Cœur d’Hérault, Béziers, France

${ }^{3}$ Service de santé au travail, Santé Travail Loire Nord, Roanne, France

${ }^{4}$ Département d'études et d'assistances médicales, Institut National de Recherche et sécurité,

Paris, France

${ }^{5}$ Unité de santé au travail, professionnelle, AP-HP UVSQ, CHU Poincaré, 92380 Garches, France

Auteur correspondant : Dr Jean-Bernard Henrotin, Département d'épidémiologie en entreprise, Institut National de Recherche et Sécurité, 1 rue du Morvan, CS 60027 Vandoeuvre-les-Nancy, 54519, Cedex, France [email: jeanbernardhenrotin@wanadoo.fr, Tel : 03. 83.50.86.12 - Fax: 03.83.50.20.15]

Mots-clés : grossesse et travail ; risques reproductifs; arrêt de travail; santé au travail; salariée enceinte Key-words : pregnancy at work; reproductive risks; sick leave; occupational health; pregnant worker 


\section{Objectif}

Décrire le suivi des grossesses au travail et les expositions professionnelles potentiellement dangereuses pour l'issue d'une grossesse.

\section{Matériels et méthodes}

Une étude descriptive transversale a été réalisée entre le $1^{\text {er }}$ janvier 2014 et le 31 décembre 2014 auprès de services de santé au travail de la région Languedoc-Roussillon. Les salariées étaient interrogées par les médecins du travail (MT) lors de la visite de reprise après accouchement (expositions professionnelles, anticipation et prévention des risques, communication entre préventeurs et cliniciens, arrêts de travail). Le codage des emplois était réalisé en utilisant la nomenclature des catégories socio-professionnelles de l'INSEE (2003). La précarité socio-économique était mesurée à partir de l'échelle EPICES.

\section{Résultats}

Dans cette étude, 1347 salariées ont été recrutées. La moyenne d'âge était de 30,7 +/- 4,5 ans. L'effectif comprenait 54,2\% d'employées, 30,7\% de professions intermédiaires, 10,4\% de cadres et $4,7 \%$ d'ouvrières. Vingt-deux pour cent des salariées présentaient une situation de précarité socio-économique. Quarante-trois pour cent de l'effectif était exposé à au moins 3 risques potentiels pour une grossesse. Seulement $17,7 \%$ des salariées avaient rencontré un MT et 14,7\% avaient bénéficié d'un aménagement de poste. Notons qu'il était rapporté un niveau élevé (74,2\%) d'arrêt de travail (AT).

\section{Conclusion}


Ces résultats descriptifs attirent l'attention sur la nécessité de surveiller les femmes enceintes au travail. Toutefois, le faible niveau d'actes de prévention et le fort niveau d'AT interrogent la façon de prendre en compte le travail dans le suivi des grossesses au travail. 


\section{Introduction}

En 2010, 70,2 \% de femmes enceintes françaises ont occupé un emploi durant leur grossesse [1]. Si travailler pendant la grossesse n'est pas une situation à risque en soi, certains lieux et contextes de travail peuvent faire que certaines femmes soient exposées à des risques pour l'évolution d'une grossesse [2, 3, 4].

Lafon et al, avaient en 2010 fait le point dans un avis d'experts sur les risques professionnels pour l'évolution d'une grossesse et les mesures de prévention utiles à mettre en place [2]. En fonction des métiers, cela peut concerner des expositions à des substances chimiques (p. ex., solvants organiques, certains métaux lourds, antimitotiques, anesthésiques gazeux ou certains pesticides) $[5,6]$ ou des expositions à un ensemble de microorganismes (p. ex., virus de la rubéole, toxoplasmose, brucella) [2, 7]. Les nuisances liées aux ambiances de travail (chaleur, froid, bruit), à la charge physique (manutention de charges lourdes, station debout prolongée, certaines postures), aux vibrations (conduite) ou à l'organisation du travail (travail de nuit) sont signalées aussi comme associées à la survenue de problèmes durant la grossesse $[2,8,9]$. Un certain nombre de ces risques peuvent faire l'objet de recommandations pour limiter leur impact sur la grossesse [2, 3, 4]. Dans le domaine des rayonnements ionisants, des effets délétères sont clairement identifiés source d'une législation protectrice $[2,10]$. Pour les radiations non ionisantes telles que les champs électromagnétiques, de nombreuses interrogations subsistent $[2,11]$.

En milieu de travail, la réglementation donne la responsabilité à l’employeur, en s'appuyant sur le service de santé au travail (SST), d'évaluer les risques ${ }^{1}$ professionnels (art. R. 4121-1 du code du travail) afin d'éviter des conséquences négatives sur la grossesse [12,

\footnotetext{
${ }^{1}$ Pour les préventeurs en santé au travail, « risque » et " danger » renvoie à deux notions différentes. Le risque est la probabilité qu'un danger provoque des effets nocifs pour la santé. Ce niveau de risque face au danger pourrait alors être modifié par la mise en place de mesures de prévention. Pour rendre la lecture du texte plus aisée, cette distinction ne sera pas systématiquement effectuée par la suite avec une utilisation privilégiée du terme " risque », plus couramment utilisé.
} 
13]. De cette évaluation doit découler une information adéquate des salariées et des mesures de prévention nécessaires pour les soustraire aux risques : restriction d'activité, d'aménagement du poste de travail, d'affectation temporaire à un autre poste, voire un arrêt de travail sous certaines conditions (risques réglementés) [4, 12, 13]. Notons également l'intérêt d'anticiper les risques pour la reproduction dès le projet de grossesse (recommandations de la Haute Autorité de santé) [14].

S'il existe des connaissances sur les dangers, des conseils de prévention possibles et une législation protectrice, l'avis d'experts soulignait l'absence de données chiffrées, dans une vision globale, sur les expositions professionnelles et les actions de prévention mise en place en entreprises concernant les grossesses au travail (GaT) [2]. C'est pourquoi, il a été mené une étude dont l'objectif était de décrire les expositions professionnelles à risque pour une grossesse avant l'accouchement et le suivi préventif des grossesses en milieu de travail (anticipation et prévention des risques, communication entre préventeurs et cliniciens, arrêts de travail).

\section{Matériels et méthodes}

\section{Population et schéma d'étude}

Une étude descriptive transversale a été réalisée du $1^{\text {er }}$ janvier 2014 au 31 décembre 2014 dans les SST de la région Languedoc-Roussillon. Les critères d'inclusion des salariées étaient les suivants: (i) avoir travaillé pendant la dernière grossesse pour un employeur; (ii) être âgé de plus de 18 ans au moment de l'accouchement (iii) bénéficié d'une visite postnatale avec le MT dans l'année suivant l'accouchement ou iv) dans les trois ans suivant 
l'accouchement, s'ils avaient eu un congé parental à plein temps; et (v) maîtriser suffisamment le français pour répondre à un questionnaire.

\section{Inclusion des femmes}

Conformément à la législation française du travail, après un accouchement et au moment de son retour au travail, chaque salariée devait bénéficier en 2014 d'une visite médicale avec un MT (articles R. 4624-21 à R 4624-23 du Code du travail). Ainsi, lors de cette première visite postnatale, les femmes ont été invitées à participer à l'étude par les MT volontaires. Basé sur une visite de reprise obligatoire pour toutes les salariées, il était attendu dans les SST un flux régulier de contacts entre salariées et médecins, s'organisant de manière aléatoire tout au long de l'année 2014. Il était donné comme consignes aux MT de proposer à toutes les femmes éligibles de participer à l'étude et ceci, sans aucune sélection (p. ex., âge, poste, activité...). Cette manière de faire, associée à une participation des MT étendue sur toute la région, portait les conditions d'un construit d'effectif représentatif de la population de salariées enceintes de cette région suivies en SST. Toutes les salariées volontaires ont donné leur consentement après information pour être incluses dans l'étude et les données étaient collectées anonymement. En 2014, il n'était pas nécessaire que cette étude soit approuvée par un comité d'éthique.

\section{Questionnaires}

Avant la visite médicale, les MT demandaient aux salariées éligibles de remplir un auto-questionnaire sur les caractéristiques sociodémographiques et le suivi de la grossesse; durant la visite, un deuxième questionnaire centré sur le travail était donné par le MT aux 
salariées. Au final, l'interrogation des salariées était basée sur la réponse à 87 questions (essentiellement en questions fermées).

\section{Données sociodémographiques}

L'âge maternel à l'accouchement, le niveau d'études, le statut marital et le nombre d'enfants à charge ont été recueillis. Les situations de précarité socioéconomique étaient repérées à I'aide de l'échelle EPICES (Evaluation de la Précarité et des Inégalités de santé dans les Centres d’Examens de Santé) [15]. Ce questionnaire a été testé en milieu obstétrique $[16,17]$ et en milieu de travail [18]. Il est structuré autour de 11 items binaires (oui/non) et la construction d'un score étendu de 0 à 100 basée sur l'addition des réponses positives. Un score $\geq$ à 30 classait la salariée en situation de précarité [15]. Également, il était de demander si les salariées avaient bénéficié d'une aide médicale à la procréation (AMP, oui / non).

\section{Données professionnelles}

Les intitulés des emplois, utilisant la nomenclature des catégories socioprofessionnelles de l'INSEE (version 2003), ont été codés par les équipes de MT et contrôlé par un codeur expérimenté [19]. Des informations sur la taille des entreprises, leur secteur d'activité professionnelle (code NAF), le type de contrat (à durée déterminée, à durée déterminée), les trajets travail-domicile (durée, mode), le temps de travail (à temps plein, à temps partiel) et l'ancienneté dans l'entreprise ont été recherchées.

\section{Risques pour la grossesse}


L'exposition en début de grossesse, à des risques potentiels pour une grossesse, a été recherchée par les MT basée sur leur connaissance des postes de travail. Dix-sept risques potentiels ont été examinés [2]: risques biologiques (travail avec de très jeunes enfants, des malades, des animaux); risques chimiques (utilisation de produits chimiques au poste de travail); travail de nuit (entre $22 \mathrm{~h} 00$ et $5 \mathrm{~h} 00$ ); risques physiques (station debout $\geq 1 \mathrm{~h}$ par jour, montée des escaliers (plusieurs fois par jour), penché en avant $\geq 1 \mathrm{~h}$ par jour, postures difficiles (membres supérieurs et/ou inférieurs), charges lourdes $\geq 5 \mathrm{~kg}$, tâches répétitives, vibrations (conduite), température $\left(<10^{\circ} \mathrm{C},>30^{\circ} \mathrm{C}\right)$, bruit $\geq 80 \mathrm{~dB}$, travaux sur machines industrielles; les rayonnements ionisants et les champs électromagnétiques. Ces variables d'exposition ont été présentées sous forme binaire (oui/non) suite à une construction en deux étapes. Une première étape, afin d'assurer une bonne discrimination des réponses, consistait à demander aux MT de répondre aux questions à partir d'une échelle de jugement de Likert à 4 modalités de réponse à choix forcé : non ; très rarement (quelques fois par mois) ; épisodiquement (quelques fois par semaine); fréquemment (quelques fois par jour). En deuxième étape, toutes ces variables étaient transformées pour les analyses en variables binaires en prenant comme référence la plus forte des situations d'exposition à savoir la réponse « oui » à la dernière modalité (fréquemment). Ainsi les modalités de repérage des expositions ont été réalisées sur un support de réponse standardisée pour l'ensemble des variables sauf pour deux variables du fait d'une réglementation spécifique en vigueur concernant ces risques $[10,12]$ : radiations ionisantes (référence oui, si réponse à l'une des 3 dernières modalités) ; travail de nuit (oui, si au moins une nuit). Enfin, une variable « nombre de risques professionnels pour la grossesse " correspondant à l'addition du nombre de réponses « oui » à ces dix-sept variables professionnelles, a été construit en quatre classes $(0,1-2,3-4, \geq 5$ risques). 


\section{Conflits famille-travail}

Plusieurs facteurs pouvant être source de conflit famille-travail à l'origine de fatigue pendant la grossesse ont été explorés [20]: les horaires de travail difficiles (long ( $\geq 8 \mathrm{~h} / \mathrm{j}$ ), irréguliers, fractionnés (pause $\geq 2 \mathrm{~h} / \mathrm{j}$ ), décalés (très tôt le matin, très tard le soir), absence de 2 jours de repos par semaine). Une variable «nombre de conflit famille-travail » a été construit en quatre classes $(0,1,2, \geq 3$ risques) en utilisant les cinq variables dichotomiques suivantes: enfants d'âge préscolaire à la maison (oui/non), trajet domicile-travail $\geq 50$ min/jour (oui/non), horaire de travail irrégulier (oui/non), absence de deux jours de repos consécutifs par semaine (oui/non).

\section{Absence au travail}

Les informations sur les arrêts de travail au cours de la grossesse ont été obtenues basées sur les questions suivantes: (i) pour chaque trimestre de la grossesse («avez-vous bénéficié d'un arrêt de travail (quelles que soient la cause et la durée) avant vos congés pathologiques ou légaux ? (oui/non) »; (ii) "avez-vous pris un arrêt de travail sans retour au travail avant des congés pathologiques ou légaux? oui/non ? (iii) Si oui, «à quel moment de votre grossesse avez-vous cessé complètement de travailler, en semaine d'aménorrhée (SA)». Les fréquences d'arrêt définitif avant la $15^{\text {ème }}$ SA et avant la $29^{\text {ème }}$ SA ont été mesurées. Dans cette étude, les trimestres étaient définis de la manière suivante: premier trimestre $<15 \mathrm{SA}$; deuxième trimestre de $15 \mathrm{SA}$ à $28 \mathrm{SA}$ inclus.

\section{Analyses statistiques}


Des tests du chi2 ont été utilisés pour comparer les variables qualitatives. La comparaison des moyennes s'appuyait sur des tests $t$ de Student. Au regard de l'impossibilité de disposer d'une base de sondage exhaustive de salariées enceintes au travail au niveau régional, la représentativité de la population étudiée a été testée en comparant les caractéristiques sociodémographiques des salariées recrutées à celles d’un effectif de salariées ayant accouché dans la région Languedoc-Roussillon intégré dans l'étude périnatalité en 2010 (données régionales non publiées [1]). Toutes les analyses ont été réalisées à l'aide du logiciel statistique Stata Software version 14.0 (Stata Corp, College Station, Texas, USA).

\section{Résultats}

Le nombre de salariées incluses dans cette étude a été de 1347. Très peu de salariées refusèrent de participer, avec seulement 22 femmes non incluses. Soixante-quinze médecins du travail (MT) ont accepté de participer (équivalent à $38 \%$ de l'effectif régional). En moyenne, 18 salariées ont été recrutées par médecin. L'inclusion s'est déroulée régulièrement sur toute l'année 2014 (entre 56 et 206 par mois). II était implanté sur 15 secteurs géographiques répartis du nord au sud de la région, intégrant toutes les grandes villes et tous les départements de la région. La proportion moyenne de données manquantes était de 1,8\% par variable avec seulement deux variables présentant une proportion de données manquantes au-dessus de $5 \%$ : précarité $(6,2 \%)$ et ancienneté au poste de travail $(6,5 \%)$

Le tableau 1 présente les caractéristiques sociodémographiques de la population d'étude en comparaison, pour certaines variables, avec les données de l'étude nationale périnatalité 2010 concernant la région Languedoc-Roussillon (données INSERM non 
publiées). Des différences plus particulières existaient pour les moins de 25 ans, les moins diplômées, les célibataires, les contrats à durée déterminée et à temps partiel. L'effectif constitué dans cette étude n'était donc pas complétement représentative de la population régionale. Vingt-deux pour cent de salariées étaient en situation de précarité socioéconomique. Dans cet effectif, $30 \%$ des salariées avaient bénéficié d’un congé parental à taux plein. Les employées et les ouvrières étaient les deux catégories utilisant plus souvent les congés parentaux après l'accouchement.

Les fréquences d'exposition à un certain nombre de facteurs de risque de conflit famille-travail sont affichées dans le tableau 2. Rapportons que $35,6 \%$ des salariées cumulaient au moins deux risques de conflit famille-travail parmi au moins 5 examinés.

Les fréquences d'exposition à 17 risques professionnels pour une grossesse sont présentées dans la figure 1. Les risques physiques étaient les expositions les plus fréquentes au poste de travail. Notons que la multi-exposition à des risques pour la grossesse était aussi une éventualité fréquente : 43,1\% des salariées étaient exposées à au moins 3 risques potentiels pour la grossesse; 27,2 \% à au moins 5 risques; $27,7 \%$ n'avaient aucune exposition signalée.

Le tableau 3 donne des informations sur le suivi en milieu de travail des salariées enceintes. Au moment du projet de grossesse, peu de salariées $(7,3 \%)$ ont évoqué avec un professionnel de santé l'impact éventuel du Travail sur la grossesse. Les contacts avec les SST étaient peu fréquents $(17,7 \%)$, notamment pour un motif en lien avec la grossesse $(3,3 \%)$, ainsi que les actes de prévention en milieu de travail (14,7\% d'aménagement de poste). Plus particulièrement, seulement $10 \%$ des salariées exposées à des produits chimiques ont bénéficié d'un bilan de dangerosité. Le dispositif réglementaire spécifique pour les femmes enceintes (travail de nuit, toxoplasmose, produits "cancérogènes, mutagènes, 
reprotoxiques ॥ ...) n'était pratiquement jamais utilisé. Enfin, les orientations pour avis entre professionnels soignants suivant médicalement la grossesse et les préventeurs de santé au travail étaient quasi-inexistantes (moins de $2 \%$ ).

La fréquence durant la grossesse « d'au moins un arrêt de travail » par trimestre (quelle qu'en soit la durée ») est présentée dans le tableau 4. Au final, 74,2 \% des salariées enceintes ont bénéficié d'au moins un arrêt de travail avant leur prise de congés pathologiques ou légaux. Également, 14 \% des salariées auront eu un arrêt de travail suivi d'aucune reprise de travail jusqu'à l'accouchement avant la fin du $1^{\text {er }}$ trimestre et 56,7\% avant la fin du $2^{\text {ème }}$ trimestre.

La figure $\mathbf{2}$ présente les fréquences « d'au moins un arrêt de travail » par trimestre et selon le nombre de risques professionnels auxquels les salariées étaient exposées. II était observé une augmentation des fréquences de survenue d'arrêt de travail au fur et à mesure de l'avancée de la grossesse et en fonction du nombre de risques auxquels étaient exposés les salariées enceinte. Pour chaque trimestre, les fréquences d'exposition entre les 4 groupes étaient significativement différentes sur le plan statistique $(p<0.001)$.

\section{Discussion}

\section{Principaux résultats}

Dans un effectif de salariées enceintes suivi en SST, la fréquence d'exposition à plusieurs risques potentiels pour une grossesse est relativement fréquente. Pour autant, il ressortait qu'il y avait peu d'actions de prévention en milieu de travail. Par contre, les fréquences d'arrêt de travail étaient conséquentes et ceci d'autant plus que les expositions professionnelles potentiellement dangereuses pour une grossesse étaient présentes. 


\section{Forces et faiblesses}

A notre connaissance, il s'agit de la première étude en France apportant, dans une vision globale, des informations quantifiées sur les expositions professionnelles et les actions de prévention dans une population de salariées enceintes. Elle a été menée sur un effectif très large, basé sur un bon taux de participation des salariées. Le recueil des expositions a été mené indépendamment des salariées. Le codage des emplois a fait l'objet d'un contrôle exhaustif. Si la nécessité de comprendre le français pouvait exposer à un biais de recrutement, aucun refus pour cette raison n'a été signalé. Un autre risque de biais de sélection a été limité en intégrant les salariées ayant bénéficié d'un congé parental à temps plein (salariées les plus modestes) [21, 22]).

Toutefois, les résultats doivent être lus au regard des informations suivantes. Elle a été menée dans une région peu industrialisée et pauvre sur le plan économique [23]. Les résultats obtenus pourraient ne valoir que pour cette région. II s'agit aussi d'une étude transversale menée après l'accouchement et des biais de sélection (p.ex., sur l'exposition) ne peuvent être exclus. Notamment, malgré les précautions prises, les salariées les plus précaires (CDD, temps partiel), vivant plus souvent seul, moins diplômées et les plus jeunes pourraient y être sous-représentées. On notera que les informations obtenues sur les arrêts de travail étaient déclaratives. Les données déclaratives comparées aux données de registres administratifs, ont tendance à sous-estimer les délais d'autant plus que les arrêts sont de durée particulièrement longue $[24,25]$. Les résultats observés dans cette étude seraient plutôt alors un minimum. Enfin, cette étude n'a intégré que les salariées suivies par des SST et n'incluait pas les salariées de très grandes entreprises et les salariées du secteur agricole 
au demeurant ultra-minoritaires compte tenu des caractéristiques économiques de la région.

\section{Interprétation}

A notre connaissance, il n'existe pas d'autres études similaires avec lesquelles cette étude pourrait être comparée. Dans la littérature, les études ont plutôt été menées dans des secteurs spécifiques (santé, soins du corps,...). La liste des risques a été construite à partir des données de l'avis de l'experts " grossesse et travail » [2, 4]. Si les liens de causalité entre santé périnatale et exposition restent discuter pour un certain nombre d'entre eux [2, 26], ces risques se présentent cependant, comme suffisamment argumentés sur le plan scientifique pour en faire des points de préoccupation dans la surveillance des grossesses et déclencher des conseils de prévention [2, 4]. Cette étude montre en premier constat que leur présence est une réalité fréquente. En cela, elle confirme que la question du Travail se présente comme une préoccupation incontournable du suivi des salariées enceintes.

En milieu de travail, les principes généraux de prévention dictés par la réglementation (L.4121-2 du Code du travail) demandent en priorité d'adapter le travail à l'homme pour protéger leur santé par la mise en place de mesures préventives et assurer le maintien au travail. Ceci est en pratique effectué par l'employeur via les SST et anticiper dans les démarches d'évaluation a priori de risques déjà évoquées (R. 4121-1 du code du travail). Evaluer et protéger des risques professionnels encourus lors d'une grossesse en fait partie [2]. Notre étude souligne clairement qu'il existe peu de contacts entre salariées et SST, et quasiment pas d'échanges entre cliniciens et préventeurs. Ainsi, les adaptations de poste ou des changements temporaires permettant le maintien au travail ont très peu l'occasion d'être organisés. Au-delà des possibilités non explorées de maintien au travail, ce 
constat peut aussi alimenter des inquiétudes sur le bon déroulement des grossesses comme illustrer par exemple par la question du risque chimique. De nos résultats, il est constaté qu'au $1^{\text {er }}$ trimestre la plupart des salariées enceintes ont été au travail (86\%). Pourtant, durant cette période, seulement $10 \%$ des salariées exposées à des produits chimiques auront bénéficié d'une évaluation de risque $[12,13]$. La possibilité qu'une exposition à certains produits chimiques puisse être délétères en tout début de grossesse est un risque clairement reconnu $[2,13]$. Notons que, l'insuffisance de prévention concernant les produits " cancérogènes, mutagènes et toxiques pour la reproduction » est un constat effectué par d'autres appelant à beaucoup plus de vigilance en la matière $[27,28]$.

La fréquence des arrêts de travail, ici à un niveau élevé $(74,2 \%)$, est assez similaire à une autre étude française de 2003 (76 \%) [1, 2] et à d'autres données européennes : 75 \% [29]; $51 \%$ [30]. Nos résultats viennent alimenter le constat en Europe d'un niveau élevé d'arrêt durant la grossesse, en constante augmentation ces dernières décennies [2, 29-34] Les publications sont sur le sujet par ailleurs peu nombreuses et les déterminants de ces arrêts restent encore à préciser [2]. Dans cette étude descriptive, il ressort que plus le nombre de risques professionnels auxquels sont exposées les salariées enceintes augmente, plus le risque d'avoir un arrêt de travail augmente. Une étude récente s'appuyant sur des analyses multivariées rapporte qu'indépendamment d'un suivi médical pour grossesse à risque, le fait d'être exposé au travail à des risques professionnels est associé à plus d'arrêts de travail, ceci d'autant plus que le nombre de risques est important [31]. Ces résultats, s'ils étaient confirmés, laissent suggérer qu'afin d'éviter les risques professionnels pendant le travail, les professionnels de santé ont plutôt recours aux arrêts de travail qu'à l'aménagement de poste de travail. 
Dans les caractéristiques de la population de cette étude, l'attention a été attirée par la fréquence non négligeable de salariées enceintes en situation de précarité socioéconomique (22\%). Cette fréquence est assez proche de celles d'autres études utilisant l'échelle EPICES menées en SST (27 \%) [18] ou en service hospitalier (25 \%) [17]. Les données de la littérature rapportent très clairement des liens entre santé périnatale et précarité mais pour des études en population générale intégrant surtout des femmes en situation de non emploi (migrantes, chômeuses) [2, 35, 36]. Qu'en est-il pour les sujets en situation de précarité au travail ? Deux études récentes confirment que cette question mérite d'être explorée. En effet, elles rapportent que ces salariées ont comme caractéristique de cumuler les expositions professionnelles à risque (notamment physique), un risque de prématurité plus important quand il y a une accumulation de risques professionnels et, un risque d'arrêts de travail plus précoces et plus longs [21,34]. Dans un contexte tels que rapporté dans cette étude où la fréquence des actes de prévention en milieu de travail semble peu fréquent, ce groupe de salariées enceintes plus particulièrement vulnérable a-t-il les possibilités ou les ressources de se protéger des risques professionnels, de recevoir les messages de prévention, les aides adéquates durant le travail et cela, au moment opportun $[2,16,17,34$, 36] ?

\section{Perspectives pratiques}

Nos résultats peuvent avoir plusieurs implications pratiques: i. ils posent ainsi l'intérêt dans la surveillance médicale des salariées enceintes de questionner systématiquement le "Travail » pour repérer ces risques dont on constate qu'ils peuvent concerner de nombreuses salariées [4] ; ii. Cette prise en compte peut obtenir le soutien des préventeurs des SST. Mais il faut pour cela que les salariées ou les employeurs (après 
déclaration de grossesse par la salariée) prennent l'initiative du contact avec le SST. En effet, il n'existe aucune procédure administrative automatique envoyant aux SST des informations sur l'état de grossesse des salariées qu'ils ont la charge de surveiller professionnellement [12] ; iii. l'information sur l'existence de dispositions spécifiques pour les femmes enceintes exposées à certains risques particuliers, déterminés par voie réglementaire, doit être mieux diffusée $[12,13]$; iv. l'organisation d'une visite très tôt en début de grossesse auprès des SST (a fortiori si l'on suspecte des risques professionnels pour la grossesse) est à encourager. Dans l'idéal, il serait souhaitable, d'évoquer l'impact éventuel du travail dès les visites préconceptionnelles $[14,26,37]$.

\section{Perspectives scientifiques}

Les résultats de cette étude reflètent la situation existante dans une région qui pourrait ne pas être représentative de la situation française. Il y a donc nécessité de répliquer ce type d'étude ailleurs. Compte tenu des enjeux sanitaires (prématurité, malformations, fausses couches...), nos résultats argumentent le besoin de disposer d'indicateurs réguliers sur le suivi professionnel des grossesses au travail. Les constats et pistes de réflexions présentés dans cette étude sont issus d'une étude descriptive transversale et en cela, devront être confirmés par d'autres études analytiques. Comme il peut exister des situations différentes selon certains groupes de salariées (e.g. en précarité socio-économique, cadres) ou selon certains secteurs d'activité (e.g. santé, soins du corps ...), des analyses ciblées par groupe ou par secteur pourraient s'avérer pertinentes également à mener. Ces études permettraient à terme de mieux informer, conseiller et orienter les patientes enceintes sur leur lieu de travail. 


\section{Conclusion}

Il ressort de cette étude que chez les salariées enceintes, l'exposition à des risques professionnels est fréquente mais associée à peu de prévention et les taux d'arrêts de travail conséquents. Les principes généraux de prévention en entreprises demandent que priorité soit faite à l'amélioration des postes de travail plutôt qu'à l'éviction des salariées du travail [2]. Sur cette base, les résultats de cette étude interrogent la façon de prendre en compte le Travail dans la surveillance des salariées enceintes. S'il existe un dispositif réglementaire en faveur de la prévention et du maintien dans l'emploi en lien avec les SST, il faut rappeler que leur implication au moment opportun, demande que les employeurs ou les salariées prennent l'initiative du contact avec ces services.

\section{Conflit d'intérêt}

Les auteurs déclarent ne pas avoir de liens d'intérêts.

\section{Remerciements}

Nous remercions particulièrement le Dr Saurel-Cubizolles MJ et Dr Blondel B de l'Institut National de la Santé et de la Recherche Médicale et responsable de l'Enquête Nationale Périnatale 2010 pour les informations spécifiques fournies concernant la région du Languedoc-Roussillon. 


\section{Références :}

[1] Blondel B, Lelong N, Kermarrec M, Goffinet F, The National Coordination Group of the National Perinatal Surveys. Trends in perinatal health in France from 1995 to 2010. Results from the French National Perinatal Surveys. J Gynecol Obstet Biol Reprod (Paris). 2012;41(4):e1-e15.

[2] Lafon D, Abadia G, Basile S, Bastide JC, Bayeux-Dunglas MC et al. Grossesse et travail. Quels sont les risques pour l'enfant à naître ? Avis d'experts. Paris : EDP Sciences ; 2010.

[3] Paul J. Partir du bon pied : guide sur la maternité sans risques et le monde du travail. Genève : Bureau International du Travail ; 2012.

[4] Servan-Schreiber E, Lafon D, Puech P, Deruelle P. Connaître les principaux risques professionnelles pour la maternité liés au travail de la mère. Rev Prat 2014;64(2):247-56.

[5] Slama R, Cordier S. Impact des facteurs environnementaux physiques et chimiques sur le déroulement et les issues de grossesse. J Gynecol Obstet Biol Reprod (Paris) 2013;42(5):413-44.

[6] Warembourg C, Cordier S, Garlantézec R. An update systematic review of fetal death, congenital anomalies, and fertility disorders among health care workers. Am J Ind Med. 2017;60(6):578-590.

[7] Morales-Suárez-Varela M, Kaerlev L, Zhu JL, Llopis-González A, Gimeno-Clemente N, et al. Risk of infection and adverse outcomes among pregnant working women in selected occupational groups: A study in the Danish National Birth Cohort. Environ Health 2010:15;9:70. 
[8] Palmer KT, Bonzini M, Harris EC, Linaker C, Bonde JP. Work activities and risk of prematurity, low birth weight and pre-eclampsia: an updated review with metaanalysis. Occup Environ Med 2013;70(4):213-22.

[9] van Beukering MD, van Melick MJ, Mol BW, Frings-Dresen MH, Hulshof CT. Physically demanding work and preterm delivery: a systematic review and meta-analysis. Int Arch Occup Environ Health 2014;87(8):809-34.

[10] Gérard JP. Occupational hazard related to ionizing radiation and surveillance of exposed people. Rev Prat 2015;65(1):90-3.

[11] Bisseriex C, Laurent P, Cabaret P, Bonnet C, Marteau E, et al. Grossesse et champs électromagnétiques (ED 4216). Paris : Institut National de Recherche et Sécurité ; 2011.

[12] Shettle J. Grossesse, maternité et travail. Aide-mémoire juridique TJ14. Paris ; Institut National de Recherche et Sécurité ; 2016.

[13] Malard S, Chapoutier-Guillon A. Femme enceinte ou allaitante exposée aux risques chimiques : aspects réglementaires. Concours médical 2015;137(7):569-571.

[14] Anonyme. Projet de grossesse : informations, messages de prévention, examens à proposer. Paris : Haute Autorité de santé ; 2009.

[15] Labbe E, Blanquet M, Gerbaud L, Poirier G, Sass C, et al. A new reliable index to measure individual deprivation: the EPICES score. Eur J Public Health 2015;25:604-9.

[16] Convers M, Langeron A, Sass C, Moulin JJ. Augier A, et al. Is the socioeconomic deprivation EPICES score useful in obstetrics ? Gynecol Obstet Fertil 2012;40:208-12.

[17] Lelong A, Jiroff L, Blanquet $M$, Mourgues $C$, Leymarie $M C$, et al. Is individual social deprivation associated with adverse perinatal outcomes? Results of a French 
multicentre cross-sectional survey. J Prev Med Hyg 2015;56:E95-E101.

[18] Moulin JJ, Labbe E, Fontana L, Charbotel B, Moulin M, et al. La santé des travailleurs en situation de vulnérabilité sociale : une expérimentation pour faciliter l'accès à la prévention et aux soins (Premtes), France, 2008-2010. Bull Epidemiol Hebd 2011;4950: $522-526$

[19] Henrotin JB, Vaissière $M$, Etaix $M$, Malard S, Dziurla $M$, et al. Codage d'emplois (PCS 2003) : retour d'expérience d’une étude menée en service de santé au travail. Sante Publique 2016;28(4):471-480.

[20] Kengatharan N. The nature of work family conflict : a review and agenda for future research. International journal of human resource studies 2015;5(2):163-188.

[21] Henrotin JB, Vaissière $M$, Etaix $M$, Dziurla $M$, Radauceanu $A$, et al. Deprivation, occupational hazards and perinatal outcomes in pregnant workers. Occup Med (Lond) 2017;67(1):44-51.

[22] Wallace $M$, Saurel-cubizolles $M-J$, and the EDEN mother-child cohort study group. Returning to work one year after childbirth: data from the mother-child cohort EDEN. Matern Child Health 2013;17(8):1432-1440.

[23] Ballet B, Bernard Nozières B, Roger Rabier R. Insee Analyses Midi-Pyrénées 2015;16:14.

[24] Voss M, Stark S, Alfredsson L, Vingard E, Josephson M. Comparisons of selfreported and register data on sickness absence among public employees in Sweden. Occup Environ Med. 2008;65(1):61-7.

[25] Ferrie JE, Kivimäki M, Head J, Shipley MJ, Vahtera J, Marmot MG. A comparison of 
self-reported sickness absence with absences recorded in employers' registers: evidence from the Whitehall II study. Occup Environ Med. 2005;62(2):74-9.

[26] Salihu HM, Myers J, August EM. Pregnancy in the workplace. Occup Med (Lond) 2012;62(2):88-97.

[27] Legrand E. Santé reproductive et travail : la prévention des risques reprotoxiques. Rapport final ANSES (REPRO $\left.-n^{\circ} 12-099\right)$ 2015. (Available from: URL: https://www.anses.fr/fr/content/sant\%C3\%A9-reproductive-et-travail-lapr\%C3\%A9vention-des-risques-reprotoxiques-2015)

[28] Ministère du travail. Plan santé-travail 2016-2020. 2017 (Available from : URL : http://travail-emploi.gouv.fr/IMG/pdf/synthese_pst3_vf.pdf )

[29] Dørheim SK, Bjordatn B, Eberhard-Gran M. Sick leave during pregnancy: a longitudinal study of rates and risk factors in a Norwegian population. BJOG 2013;120(5):521-530.

[30] Kristensen P, Nordhagen R, Wergeland E, Bjerkedal T. Job adjustment and absence from work in mid-pregnancy in the Norwegian Mother and Child Cohort Study (MoBa). Occup Environ Med 2008;65(8):560-6.

[31] Henrotin JB, Vaissière $M$, Etaix $M$, Dziurla $M$, Radauceanu $A$, et al. Exposure to occupational hazards for pregnancy and sick leave in pregnant workers: a crosssectional study. Ann Occup Environ Med 2017;29:12.

[32] Alexanderson K, Hensing G, Carstensen J, Bjurulf P. Pregnancy-related sickness absence among employed women in a Swedish county. Scand J Work Environ Health. 1995;21(3):191-8. 
[33] Kaerlev L, Jacobsen LB, Olsen J, Bonde JP. Long-term sick leave and its risk factors during pregnancy among Danish hospital employees. Scand J Public Health. 2004;32(2):111-7.

[34] Vigoureux S, Blondel B, Ringa V, Saurel-Cubizolles MJ. Occupational, social and medical characteristics of early prenatal leave in France. Eur J Public Health 2016;26(6):1022-1027.

[35] Mahieu-Caputo D. Prise en charge des femmes enceintes et nouveau-nés en situation de vulnérabilité sociale : faut-il une prise en charge spécifique ? Gynecol Obstet Fertil 2010;38:83-91.

[36] Le Méner E. L'impact de la précarité sur la santé des femmes enceintes. La santé en action 2016;437:26-27.

[37] Bretelle F. Consultation préconceptionnelle : pour évaluer le risque et orienter. Concours médical 2010;132(8): 330-332. 
Tableau 1 : comparaison des caractéristiques sociodémographiques de l'effectif GaT 2014 à celui de l'étude périnatalité 2010 du même secteur géographique†

\begin{tabular}{|c|c|c|}
\hline Variables & $\begin{array}{l}\text { GaT } \\
\mathbf{2 0 1 4} \\
n=1347\end{array}$ & $\begin{array}{l}\text { Périnat } \\
\text { 2010t } \\
n=342\end{array}$ \\
\hline \multicolumn{3}{|l|}{ Age maternel } \\
\hline Moyenne (ans, et) & $30,7(4,5)$ & $30,3(5,35)$ \\
\hline$<25$ ans $(n, \%)^{* * *}$ & $122(9,2)$ & $58(15,6)$ \\
\hline \multicolumn{3}{|l|}{ Formation } \\
\hline$\leq$ baccalauréat $(n, \%)^{*}$ & $495(37,1)$ & $150(40,6)$ \\
\hline \multicolumn{3}{|l|}{ Groupes sociaux $(n, \%) \$$} \\
\hline Agriculture (salariées) & $0(0)$ & $10(2,7)$ \\
\hline Cadres, prof scientifiques & $140(10,4)$ & $41(11,0)$ \\
\hline Prof intermédiaires & $413(30,7)$ & $97(26,2)$ \\
\hline Employées & $729(54,2)$ & $199(53,6)$ \\
\hline Ouvrières & $63(4,7)$ & $24(6,5)$ \\
\hline \multicolumn{3}{|l|}{ Vie en couple } \\
\hline Non $(n, \%)^{*}$ & $53(3,8)$ & $23(6,2)$ \\
\hline \multicolumn{3}{|l|}{ Aucun enfant à charge $¥$} \\
\hline Oui $(n, \%)^{*}$ & $719(53,6)$ & $192(52,0)$ \\
\hline \multicolumn{3}{|l|}{ Aide médicale à la procréation } \\
\hline Oui $(n, \%)$ & $86(6,4)$ & $29(7,9)$ \\
\hline \multicolumn{3}{|l|}{ Temps de travail } \\
\hline Temps partiel** & $258(19,4)$ & $96(26,5)$ \\
\hline \multicolumn{3}{|l|}{ Contrat de travail } \\
\hline A durée déterminée ${ }^{* * *}$ & $62(4,7)$ & $71 \quad(19,1)$ \\
\hline \multicolumn{3}{|l|}{ Ancienneté au poste de travail } \\
\hline Moyenne (ans, et) & $4,8(3,6)$ & - \\
\hline$<2$ ans $(n, \%)$ & $300(22,5)$ & - \\
\hline Période d'essai & & - \\
\hline Oui $(n, \%)$ & $56(4,2)$ & \\
\hline \multicolumn{3}{|l|}{ Multi-employeur } \\
\hline Oui $(n, \%)$ & $80(6,0)$ & - \\
\hline \multicolumn{3}{|l|}{ Précarité socio-économique } \\
\hline Oui, score EPICES $\geq 30(n, \%)$ & $277(22,0)$ & - \\
\hline \multicolumn{3}{|l|}{ p-value : ${ }^{*} p<0.05 * p<0.01 * * * p<0.001$} \\
\hline \multicolumn{3}{|c|}{ \$ : p>0.05 sans secteur agricole (non suivi en service interentreprises) } \\
\hline \multicolumn{3}{|c|}{$\mathrm{P}<0.001$ avec secteur agricole } \\
\hline \multicolumn{3}{|c|}{ †Départements français concernés : $48,30,34,11,66,84$ ¥pendant la grossesse } \\
\hline \multicolumn{3}{|c|}{ et: ecart-type $\quad \mathbf{n}$ : nombre $\quad$ GaT : étude Grossesse au Travail } \\
\hline \multicolumn{3}{|c|}{$\begin{array}{l}\text { Périnat } 2010 \text { : données régionales de l'étude nationale périnatalité } 2010 \\
\text { [INSERM, données non publiées] }\end{array}$} \\
\hline
\end{tabular}


Tableau 2. Fréquences d'exposition à des sources potentielles de conflit famille-travail

\begin{tabular}{|c|c|}
\hline & $\begin{array}{l}\text { Étude GaT } 2014 \\
(n=1347)\end{array}$ \\
\hline \multicolumn{2}{|l|}{ Horaires difficiles } \\
\hline Fractionné, pause $\geq 2 \mathrm{~h} / \mathrm{j}(n, \%)$ & $307(23,2)$ \\
\hline Durée longue, $\geq 8$ h par jour ( $n, \%)$ & $266(20,0)$ \\
\hline Irrégulier (n,\%) & $423(31,6)$ \\
\hline Décalés $(n, \%)$ & $297(22,3)$ \\
\hline Sans 2 jours de repos consécutif ( $n, \%$ ) & $332(25,3)$ \\
\hline \multicolumn{2}{|l|}{ Transport } \\
\hline Mode de transport (voiture) & $1238(92,9)$ \\
\hline Durée $\geq 50 \mathrm{~min} / \mathrm{j}(n, \%)$ & $302(23,2)$ \\
\hline Médiane (min/j) & 30 \\
\hline Ressenti pénible $(n, \%)$ & $272(20,7)$ \\
\hline \multicolumn{2}{|l|}{ Enfant non scolarisé à charge } \\
\hline Oui $(n, \%)$ & $318(23,7)$ \\
\hline \multicolumn{2}{|l|}{ Nombre conflits famille-travail* } \\
\hline Aucun $(n, \%)$ & $320(25,1)$ \\
\hline 1 risque $(n, \%)$ & $500(39,3)$ \\
\hline 2 risques $(n, \%)$ & $320(25,1)$ \\
\hline$\geq 3$ risques $(n, \%)$ & $133(10,5)$ \\
\hline
\end{tabular}


Tableau 3. Suivi des salariées enceintes et travail (anticipation, professionnel, communication, prévention)

Etude GaT 2014

$(n=1347)$

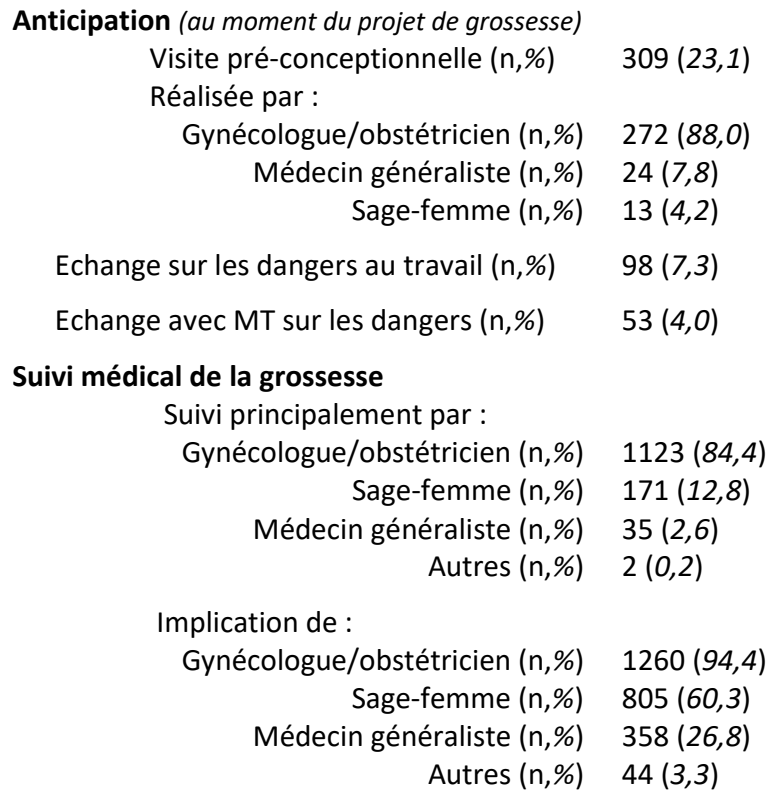

MT: médecin du travail GaT : Grossesse au Travail n: nombre 
Tableau 4. Arrêts de travail durant la grossesse (selon la déclaration des salariées)

\section{Etude GaT 2014}

$(n=1347)$

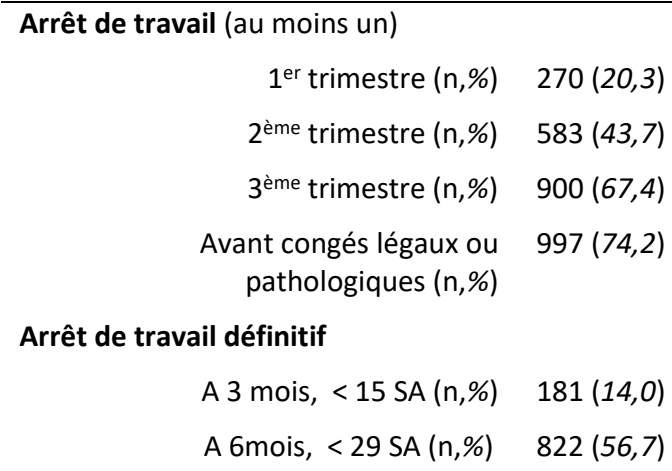

GaT : Grossesse au Travail n: nombre

SA : semaine d'aménorrhée 


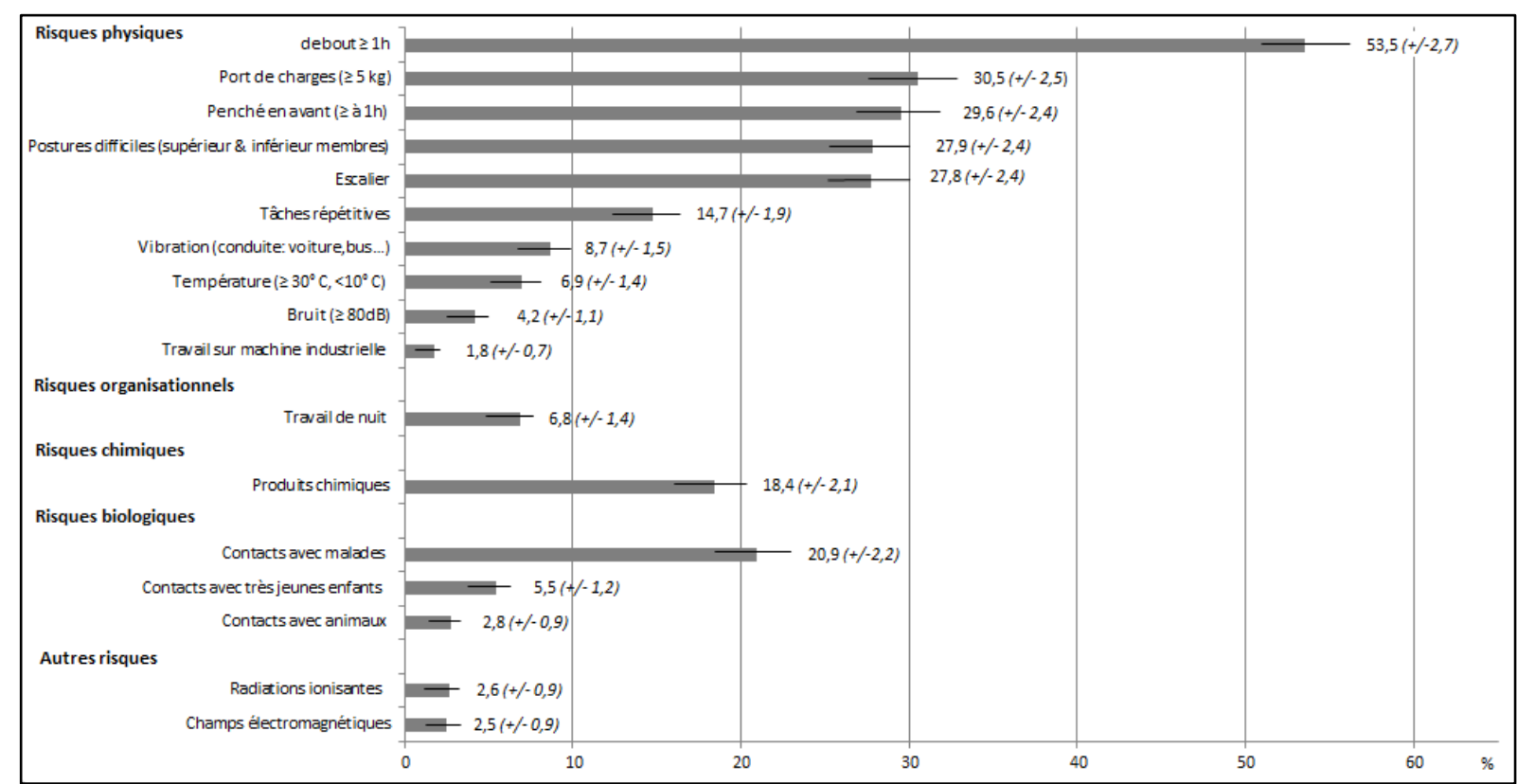

Figure 1. Fréquences d'exposition à des risques professionnels pour une grossesse (\%, intervalles de confiance à $95 \%)$ dans un effectif de salariées enceintes suivi en services interentreprises de santé au travail (étude GaT 2014, région LanguedocRoussillon). 


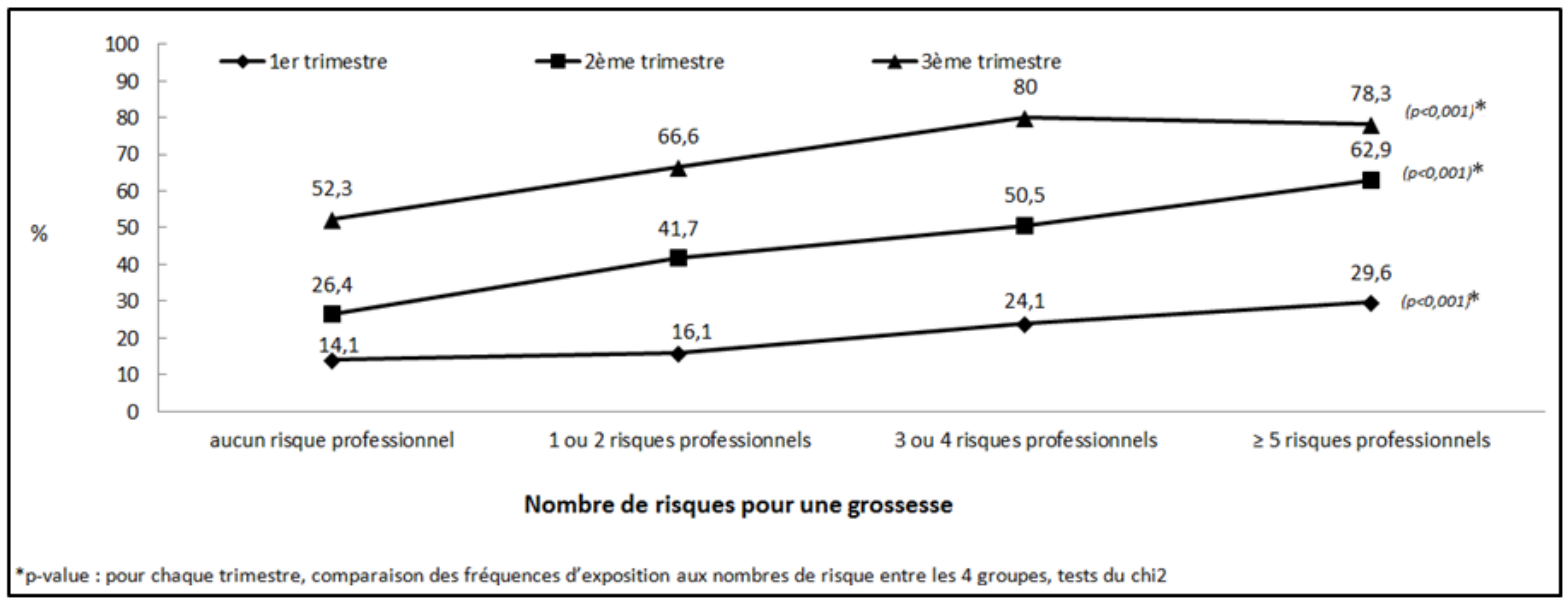

Figure 2. Fréquence "d'au moins un arrêt de travail " par trimestre et selon le nombre de risques professionnels auxquels étaient exposées les salariées enceintes (Etude GaT 2014, région Languedoc-Roussillon). 